\title{
BOUNDED SPIRAL-LIKE FUNCTIONS WITH FIXED SECOND COEFFICIENT
}

\author{
M.K. AOUF \\ Department of Mathematics \\ Faculty of Science \\ University of Mansoura \\ Mansoura, Egypt \\ (Received July 10, 1986)
}

ABSTRACT. Let $F_{p}(\alpha, \beta, M)\left(0<p<1,|\alpha|<\frac{\pi}{2}, 0<\beta<1\right.$ and $\left.M>1 / 2\right)$, denote the class of functions $f(z)$ which are regular in $U=\{z:|z|<1\}$ and of the form $f(z)=z+\left|a_{2}\right| e^{-1 \alpha} z^{2}+\ldots$, where $\left|a_{2}\right|=p(1+\sigma)(1-\beta) \cos \alpha$, which satisfy for fixed $M, z=r e^{i \theta} \varepsilon U$ and

$$
\left|\frac{e^{1 \alpha} \frac{z f^{\prime}(z)}{f(z)}-\beta \cos \alpha-1 \sin \alpha}{(1-\beta) \cos \alpha}-M\right|<M .
$$

In this paper we have found the sharp radius of $\gamma$-spiralness of the functions belonging to the class $F_{p}(\alpha, \beta, M)$.

KEY WORDS AND PHRASES. Spirallike, bounded functions, radius of $\gamma$-spiralness. • 1980 AMS CLASSIFICATION CODES. 30A32, 30A36.

1. INTRODUCTION. Let A denote the class of functions which are regular and univalent in the unit disc $U=\{z:|z|<1\}$ and satisfy the conditions $f(0)=0=f^{\prime}(0)-1$.

Let $F(\alpha, \beta, M)\left(|\alpha|<\frac{\pi}{2}, 0<\beta<1\right.$ and $\left.M>1 / 2\right)$ denote the class of bounded $\alpha$-spirallike $f$ unctions of order $\beta$, that is $f \in F(\alpha, \beta, M)$ if and only if for $f$ ixed $M$,

$$
\left|\frac{e^{i \alpha} \frac{z f^{\prime}(z)}{f(z)}-\beta \cos \alpha-i \sin \alpha}{(1-\beta) \cos \alpha}-M\right|<M, z \in U
$$

The class $F(\alpha, \beta, M)$ introduced by Aouf [1], he proved that if $f(z)=z+a_{2} z^{2}+\ldots \varepsilon F(\alpha, \beta, M)$ then,

$$
\left|a_{2}\right|<(1+\sigma)(1-\beta) \cos \alpha, \sigma=1-\frac{1}{M} .
$$


If $\varepsilon=\exp \left(-1 \arg a_{2}-i \alpha\right)$, then $\frac{f(\varepsilon z)}{\varepsilon}=z+\left|a_{2}\right| e^{-i \alpha_{z}{ }^{2}}+\ldots \varepsilon F(\alpha, \beta, M)$, whenever $f(z) \in F(\alpha, \beta, M)$. Thus without loss of generality we can replace the second coefficient $a_{2}$ of $f(z) \in F(\alpha, \beta, M)$ by $\left|a_{2}\right| e^{-1 \alpha}$.

Let $F_{p}(\alpha, \beta, M)$ denote the class of functions $f(z)=z+\left|a_{2}\right| e^{-1 \alpha_{z} 2}+\ldots$ which satisfy (1.1), where $\left|a_{2}\right|=p(1+\sigma)(1-\beta) \cos \alpha$. In view of (1.2) it follows that $0<p<1$.

Let $G_{p}(\alpha, \beta, M)$ denote the class of functions $g(z)=z+\left|b_{2}\right| e^{-1 \alpha_{z} 2}+\ldots$, regular in $U$ and satisfy the condition

$$
\left|\frac{e^{1 \alpha}\left(1+\frac{z g^{\prime \prime}(z)}{g^{\prime}(2)}\right)-\beta \cos \alpha-1 \sin \alpha}{(1-\beta) \cos \alpha}-M\right|<M, z \in U,
$$

where $\left|b_{2}\right|=\frac{1}{2} p(1+\sigma)(1-\beta) \cos \alpha$.

It follows from (1.1) and (1.3) that

$$
g(z) \& G_{p}(\alpha, \beta, M), \text { if and on } 1 y \text { if } z g^{-}(z) \in F_{p}(\alpha, \beta, M) \text {. }
$$

We note that by giving specific values to $p, \alpha, \beta$ and $M$, we obtain the following important subclasses studied by various authors in earlier papers:

(1) $F_{1}(\alpha, \beta, M)=F_{M}(\alpha, \beta)$ and $G_{1}(\alpha, \beta, M)=G_{M}(\alpha, \beta)$, are respectively the class of bounded splrallike functions of order $B$ and the class of bounded Robertson functions of order $B$ investigated by Aouf $[1]$ and $F_{1}(\alpha, 0, M)=F_{\alpha, M}$ and $G_{1}(\alpha, 0, M)=G_{\alpha, M}$, are respectively the class of bounded spirallike functions and the class of bounded Robertson functions investigated by Kulshrestha [2] .

(i1) $F_{p}(\alpha, \beta, \infty)=F_{p}(\alpha, \beta)$ and $G_{p}(\alpha, \beta, \infty)=G_{p}(\alpha, \beta$,$) , are considered by Umaran 1$

In this paper we determine the sharp radius of $\gamma$-spiralness of the functions belonging to the class $F_{p}(\alpha, \beta, M)$, generalizing an earlier result due to Kulshrestha [2], Libera [4], Umaran1 $[5,3]$.

The technique employed to obtain this result is similar to that used by McCarty [6] and Umarani [3].

2. THE SHARP RADIUS OF $\gamma$-SPIRALNESS OF THE CLASS $F_{p}(\alpha, \beta, M), M>1$.

LEMMA 1. If $f(z) \in F_{p}(\alpha, \beta, M) M>1$, then $\left|\frac{z f^{-}(z)}{f(z)}-w_{0}\right|<\rho_{0}$, 
where

$$
w_{0}=\frac{(1+p r)^{2}+\left\{\left[(1-\beta)\left(\frac{1+\sigma}{\sigma}\right)-1\right] \cos \alpha-i \sin \alpha\right\} e^{-i \alpha} r^{2}(r+p)^{2}}{\left(1-r^{2}\right)\left(1+2 p r+r^{2}\right)}
$$

and

$$
\rho_{0}=\frac{(1+\sigma)(1-\beta) \cos \alpha r(1+p r)(r+p)}{\left(1-r^{2}\right)\left(1+2 p r+r^{2}\right)}
$$

This result is sharp.

PROOF. Let $f(z) \in F_{p}(\alpha, \beta, M), M>1$, then there exists a function $w(z)$ analytic in $U$ and $|w(z)|<1$ in $U$ such that

$$
e^{i \alpha} \frac{z f^{\prime}(z)}{f(z)}=\cos \alpha\left\{\frac{1+\left[(1-\beta)\left(\frac{1+\sigma}{\sigma}\right)-1\right] \sigma w(z)}{1-\sigma w(z)}\right]+1 \sin \alpha, \sigma=1-\frac{1}{M}
$$

or

$$
\frac{z f^{-}(z)}{f(z)}=\frac{1+\left\{\left[(1-\beta)\left(\frac{1+\sigma}{\sigma}\right)-1\right] \cos \alpha-1 \sin \alpha\right\} e^{-i \alpha} \sigma w(z)}{1-\sigma w(z)} .
$$

Solving for $w(z)$,

$$
w(z)=\frac{\frac{z f^{\prime}(z)}{f(z)}-1}{\left.\sigma\left[\frac{z f^{\prime}(z)}{f(z)}+\left\{[1-\beta)\left(\frac{1+\sigma}{\sigma}\right)-1\right] \cos \alpha-1 \sin \alpha\right\} e^{-1 \alpha}\right]} .
$$

Since $f(z)=z+\left|a_{2}\right| e^{-i \alpha_{z}{ }^{2}}+\ldots$, we obtain $w(z)=p z+\ldots=z \phi(z)$, where $\phi(z)$

is analytic in $U, \phi(0)=p$ and $|\phi(z)|<1$ in U. Now $\frac{\phi(z)-p}{1-p \phi(z)} \quad z$. Therefore

$$
\phi(z) \quad \frac{z+p}{1+p z} \text {. Also }|w(z)|=|z \phi(z)|<\frac{|z|+p}{1+|z| p}|z| \text {. Let } g(z)=\frac{|z|+p}{1+p|z|} z
$$

and

$$
h(z)=\frac{1+\left\{\left[(1-\beta)\left(\frac{1+\sigma}{\sigma}\right)-1\right] \cos \alpha-i \sin \alpha\right\} e^{-1 \alpha} \sigma z}{1-\sigma z} .
$$

Since the image of $|z|<r$ under $g(z)$ is a disc and $h(z)$ is a bilinear transformation, then $\frac{z f^{\prime}(z)}{f(z)}$ is subordinate to (hog) (z). That is, the image of $|z|<r$ under $\frac{z f^{\prime}(z)}{f(z)}$ is contained in the image of $|z|<r$ under (hog)(z).

Equality in (2.1) can be attained by a function

$$
f(z)=z\left(1-2 p \sigma z+\sigma z^{2}\right)^{-\left(\frac{1+\sigma}{2 \sigma}\right)(1-\beta) \cos \alpha e^{-i \alpha}}
$$


$=z+p(1+\sigma)(1-\beta) \cos \alpha e^{-i \alpha z^{2}+\ldots} \cdot ;$

hence

$$
\begin{aligned}
\frac{z f^{\prime}(z)}{f(z)} & =\frac{1-2 p \sigma z+\sigma z^{2}-(1+\sigma)(1-\beta) \cos \alpha e^{-1 \alpha} z(z-p)}{1-2 p \sigma z+\sigma z^{2}} \\
& =\frac{1+\sigma \psi-(1+\sigma)(1-\beta) \cos \alpha e^{-1 \alpha} \psi}{1+\sigma \psi},
\end{aligned}
$$

where $\psi=\frac{z(z-p)}{1-p \sigma z}$.

Since $p<1,0<\sigma<1,|\psi|<1$ for $z \in U$.

This shows that

$$
e^{i \alpha} \frac{z f^{\prime}(z)}{f(z)}=\cos \alpha\left\{\frac{1+\left[1-\left(\frac{1+\sigma}{\sigma}\right)(1-\beta)\right] \sigma \psi(z)}{1+\sigma \psi(z)}\right\}+1 \sin \alpha
$$

and

$$
\frac{e^{i \alpha \frac{z f^{\prime}(z)}{f(z)}-1 \sin \alpha-\beta \cos \alpha}}{(1-\beta) \cos \alpha}=\frac{1-\psi(z)}{1+\sigma \psi(z)}
$$

Then it is easy to show that $\left|\frac{1-\psi(z)}{1+\sigma \psi(z)}-M\right|<M, \sigma=1-\frac{1}{M}$. Thus $f \varepsilon F(\alpha, B, M)$.

Substituting $\psi=-\frac{\delta\left(\delta-\sigma e^{i \alpha}\right)}{\sigma\left(1-\sigma \delta e^{i \alpha}\right)}$, where $\delta=\frac{r(r+p)}{1+r p}$ in (2.5), we find that

$\left|\frac{z f^{\prime}(z)}{f(z)}-w_{0}\right|=\rho_{0}$, where $w_{0}$ and $\rho_{0}$ are given by (2.2) and (2.3).

This completes the proof of the lemma.

\section{REMARK 1.}

(1) If $p=1$ and $B=0$ in Lemma 1, we obtain a result of Kulshrestha [2].

(ii) If $M=\infty(\sigma=1)$ in Lemma 1, we obtain a result of Umarani [3].

(1ii) If $\alpha=0$ and $M=\infty(\sigma=1)$ in Lemma 1 , we obtain a result of McCarty [6].

THEOREM 1. If $f(z) \in F_{p}(\alpha, \beta, M),>1$, then $f(z)$ is $\gamma$-spiral

$|z|<r_{\gamma}$, where $r_{\gamma}$ is the smallest positive root of the equation

$$
\begin{aligned}
& \cos \gamma+p[2 \cos \gamma-(1+\sigma)(1-\beta) \cos \alpha] r+ \\
& {\left[p^{2} \cos \gamma+c p^{2}-(1+\sigma)(1-\beta) \cos \alpha\left(1+p^{2}\right)\right] r^{2}} \\
& +p[2 c-(1+\sigma)(1-\beta) \cos \alpha] r^{3}+c r^{4}=0,
\end{aligned}
$$


where $c=\cos (\gamma-2 \alpha)+\left[(1-\beta)\left(\frac{1+\sigma}{\sigma}\right)-2\right] \cos \alpha \cos (\gamma-\alpha)$. The result is sharp. PROOF. Let $f(z) \quad \varepsilon F_{p}(\alpha, \beta, M), M>1$, then by the above Lemma, we have

$$
\left|\frac{z f^{\prime}(z)}{f(z)}-w_{0}\right|<\rho_{0}
$$

Hence $\operatorname{Re} e^{i \gamma} \frac{z f^{\prime}(z)}{f(z)}>\operatorname{Re} e^{i \gamma} \cdot w_{0}-\rho_{0}$

$\cos \gamma(1+p r)^{2}+\operatorname{Re}\left\{\left[(1-\beta)\left(\frac{1+\sigma}{\sigma}\right)-1\right] \cos \alpha-1 \sin \alpha\right\} e^{i(\gamma-\alpha)} r^{2}(r+p)^{2}$

$=\frac{-(1+\sigma)(1-\beta) \cos \alpha r(1+p r)(r+p)}{\left(1-r^{2}\right)\left(1+2 p r+r^{2}\right)}$

$\cos \gamma(1+p r)^{2}+\left\{\cos (\gamma-2 \alpha)+\left[(1-\beta)\left(\frac{1+\sigma}{\sigma}\right)-2\right] \cos \alpha \cos (\gamma-\alpha)\right\} r^{2}(r+p)^{2}$

$=\left[\frac{-(1+\sigma)(1-\beta) \cos \alpha r(1+p r)(r+p)}{\left(1-r^{2}\right)\left(1+2 p r+r^{2}\right)}\right]$.

$f(z)$ is $\gamma$-spiral if the R.H.S. of (2.7) is positive. Hence $f(z)$ is $\gamma$-spiral for $|z|<r_{\gamma}$ where $r_{\gamma}$ is the smallest positive root of the equation

$\cos \gamma(1+p r)^{2}+\left\{\cos (\gamma-2 \alpha)+\left[(1-\beta)\left(\frac{1+\sigma}{\sigma}\right)-2\right] \cos \alpha \cos (\gamma-\alpha)\right\} \cdot r^{2}(r+p)^{2}$

$-(1+\sigma)(1-\beta) \cos \alpha r(1+p r)(r+p)=0$.

Simplifying the above equation, we obtain (2.6).

If $\gamma=0$ in the above theorem, we obtain the radius of starlikeness of the class $F_{p}(\alpha, \beta, M)$.

COROLLARY 1. $f(z) \in F_{p}(\alpha, \beta, M), M>1$, is starlike for $|z|<r_{0}$, where $r_{0}$ is the least positive root of the equation

$$
\begin{aligned}
& 1+p[2-(1+\sigma)(1-\beta) \cos \alpha] r+ \\
& \left(\frac{1+\sigma}{\sigma}\right)(1-\beta) \cos \alpha\left[\cos \alpha p^{2}-\sigma\left(1+p^{2}\right)\right] r^{2}+ \\
& p[2 c-(1+\sigma)(1-\beta) \cos \alpha] r^{3}+c r^{4}=0
\end{aligned}
$$

where $c=\left(\frac{1+\sigma}{\sigma}\right)(1-\beta) \cos ^{2} \alpha-1$.

If $p=1, \gamma=0$ and $\beta=0$ in Theorem 1, we obtain a result of Kulshrestha [2].

COROLLARY 2. $f(z) \in F_{\alpha, M}, M>1$, is starlike for $|z|<r_{0}$, where $r_{0}$ is the least positive root of the equation

$$
1-(1+\sigma) \cos \alpha r+\left[\left(\frac{1+\sigma}{\sigma}\right) \cos ^{2} \alpha-1\right] r^{2}=0
$$


REMARK 2.

(1) If $M=\infty(\sigma=1)$ in Theorem 1, we obtain a result of Umarani [3].

(11) If $p=1$ and $M=\infty \quad(\sigma=1)$ in Theorem 1, we obtain a result of Libera [4] and Umaran1 [5].

(1ii) If $p=1, \beta=0, \quad \gamma=0$ and $M=\infty \quad(\sigma=1)$ in Theorem 1 , we obtain a result of Robertson [7].

Since $g(z) \in G_{p}(\alpha, \beta, M)$ if and only if $2 g^{\circ}(z) \in F_{p}(\alpha, \beta, M)$ we obtain from Theorem 1 ,

THEOREM 2. If $g(z) \in G_{p}(\alpha, \beta, M), M>1$, then $\operatorname{Re} e^{i \gamma}\left(1+\frac{z g^{\prime \prime}(z)}{g^{\prime}(z)}\right)>0$ for

$|z|<r_{\gamma}$, where $r_{\gamma}$ is the least positive root of equation (2.6).

The result is sharp.

If $\gamma=0$ in Theorem 2, we obtain the radius of convexity of the class $G_{p}(\alpha, \beta, M)$.

COROLLARY 3. If $g(z) \in G_{p}(\alpha, \beta, M), M>1$, then the radius of convexity of $g(z)$ is the least positive root of equation (2.8).

REMARK 3.

(1) For $M=0(\sigma=1)$ in Theorem 2, and Coro11ary 3, we obtain a results of Umarani [3].

(11) If $p=1$ and $B=0$ in Corollary 3, we obtain a result of Kulshrestha [2]

(111) For $p=1$ and $M=\infty(\sigma=1)$, Theorem 2, generalizes the result of Umarani [5].

\section{REFERENCES}

1. AOUF, M.K. Bounded p-valent Robertson functions of order $\alpha$, Ind1an J. of Pure and App1. Maths., 16 (1985), 775-790.

2. KULSHRESTHA, P.K. Bounded Robertson functions, Rend. di Matemat1ca, (6)9, (1976), 137-150.

3. UMARANI, P.G. Spiral-1ike functions with fixed second coefficient, Indian J. Pure app1. Math. 13(3) (1982), 370-374.

4. LIBERA, R.J. Univalent a-spiral functions, Canad. J. Math. 19 (1967), 449-456.

5. UMARANI, P.G. Some studies in univalent functions, Ph.D. Thesis, Karnataka University, Dharwad (1976).

6. McCARTY, C.P. Two radius of convexity problems, Proc. Amer. Math. Soc. 42 (1972), 153-160.

7. ROBERTSON, M.S. Radil of starlikeness and close-to-convexity, Proc. Amer. Math. Soc. 16 (1965), 847-852. 


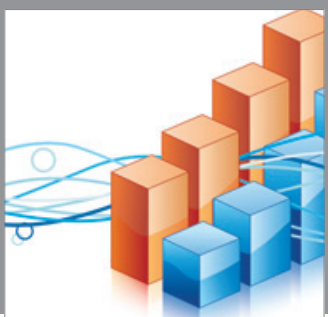

Advances in

Operations Research

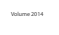

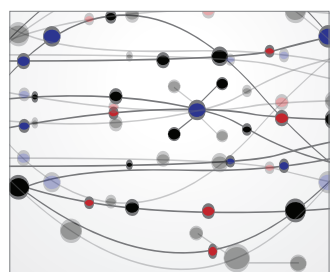

\section{The Scientific} World Journal
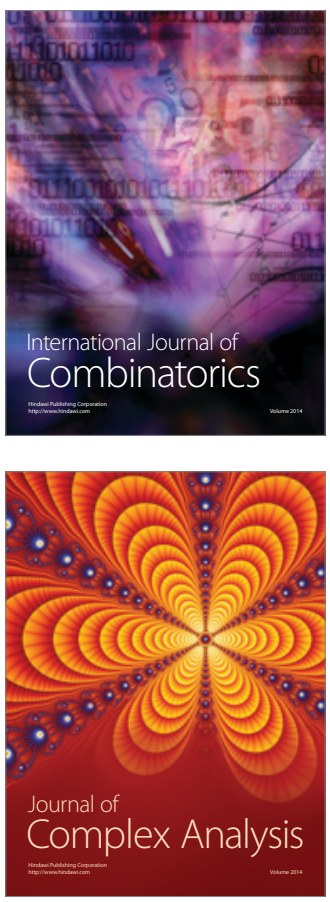

International Journal of

Mathematics and

Mathematical

Sciences
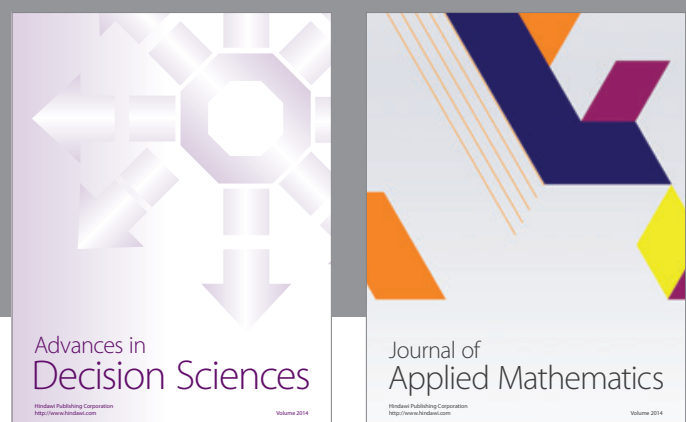

Journal of

Applied Mathematics
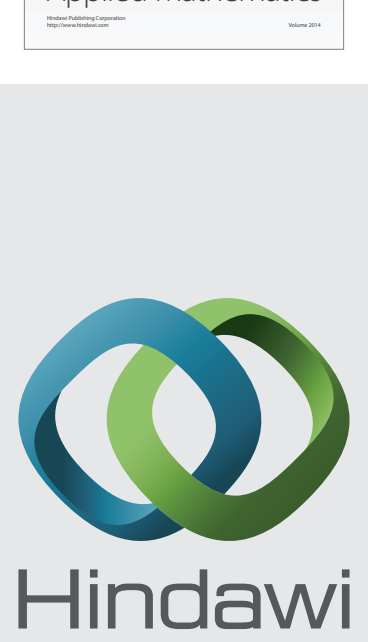

Submit your manuscripts at http://www.hindawi.com
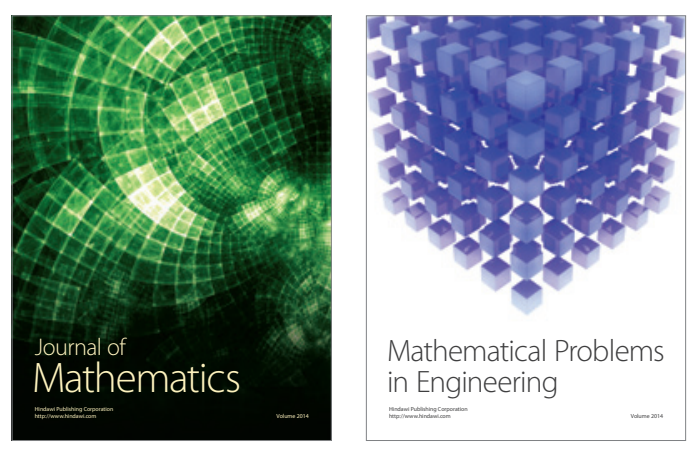

Mathematical Problems in Engineering
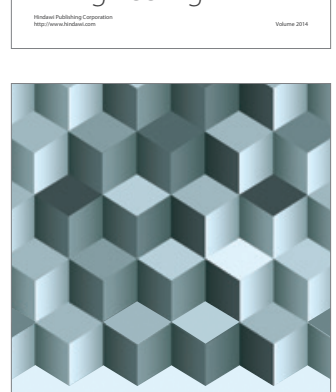

Journal of

Function Spaces
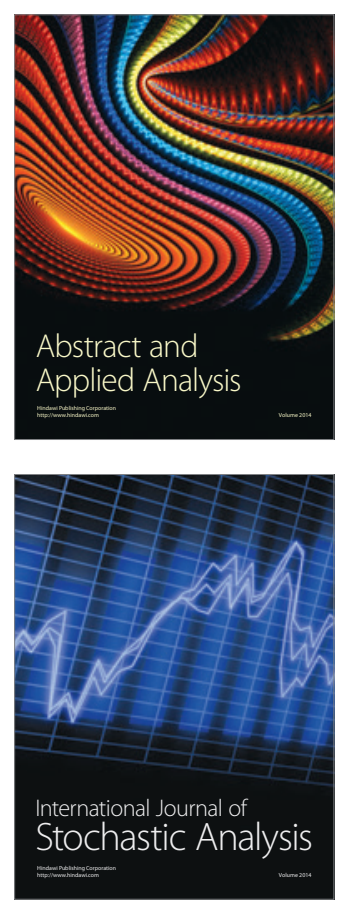

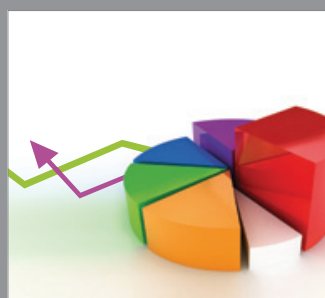

ournal of

Probability and Statistics

Promensencen
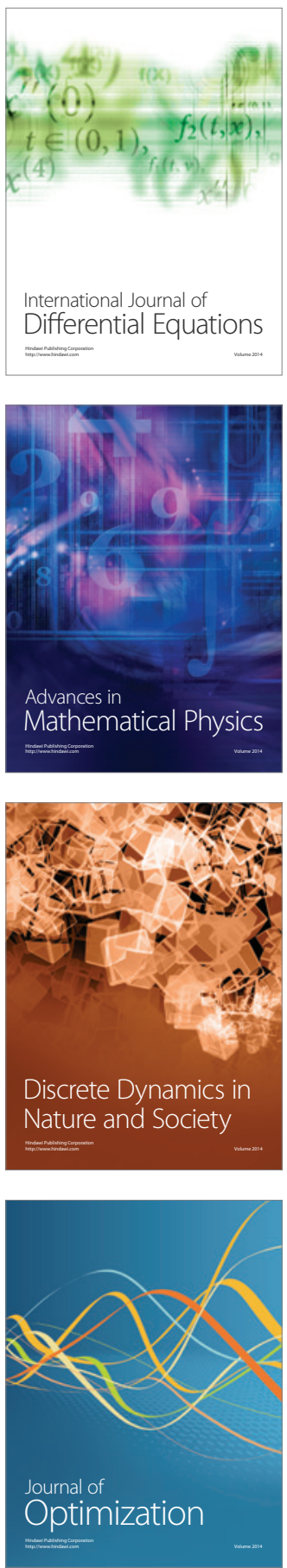